\title{
The advantage of simple symbols for learning and transfer
}

\author{
VLADIMIR M. SLOUTSKY, JENNIFER A. KAMINSKI, and ANDREW F. HECKLER \\ Ohio State University, Columbus, Ohio
}

\begin{abstract}
A goal of successful learning is the transfer of learned knowledge to novel situations. However, spontaneous transfer is notoriously difficult to achieve. In this research, we argue that learning and transfer can be facilitated when knowledge is expressed in an abstract, generic form. In Experiments 1 and 2 , undergraduate students learned two isomorphic domains, which were based on the same algebraic group, with one domain expressed in a more abstract, generic form and the other expressed in a more concrete form. In both experiments, transfer from more abstract to more concrete was greater than the reverse. In Experiment 3, undergraduate students learned the same algebraic group under varying degrees of concreteness. Our results demonstrate that the use of perceptually rich, concrete symbols may hinder learning. This research indicates that concreteness may have substantial learning and transfer costs, whereas abstractness may have benefits.
\end{abstract}

A goal of successful learning is transfer, or the ability to apply acquired knowledge outside of the learned situation. For example, if one learned how to calculate the probability of heads occurring twice on two fair coin tosses in a mathematics classroom, one should be able to apply this knowledge to the biology problem of calculating the probability that a recessive trait would be manifested in two offspring individuals. However, spontaneous transfer is notoriously difficult to achieve, even for relatively simple knowledge (Detterman, 1993). What factors hinder or facilitate transfer?

A deeply entrenched and widely held belief in the education community has been that learning and transfer can be facilitated through the use of concrete materials, which include both physical manipulatives and concrete instantiations of abstract concepts. For example, $84 \%$ of surveyed secondary school mathematics teachers expressed a belief that such concrete materials have facilitative effects on learning (Howard, Perry, \& Tracey, 1997).

However, it seems likely that facilitative effects of concreteness are limited to cases in which concrete representations communicate relevant aspects of the to-be-learned information (for a review, see Goldstone \& Sakamoto, 2003). For example, two closed and connected containers with a fixed amount of fluid, which can freely flow between the containers, may more easily communicate the idea of two players involved in a zero-sum game than the equation $x+y=\mathrm{K}$. Similarly, Dienes blocks (Dienes, 1960) can communicate the idea of the base 10 number

This research has been supported by a grant from the National Science Foundation (REC 0208103) to V.M.S. We thank Anna Fisher and Chris Robinson for helpful comments. Correspondence should be addressed to V. M. Sloutsky, Center for Cognitive Science, 208C Ohio Stadium East, 1961 Tuttle Park Place, Ohio State University, Columbus, OH 43210 (e-mail: sloutsky.1@osu.edu). system, thus possibly facilitating learning of the system. However, even if this "relevant concreteness" facilitates learning, its effects on transfer are questionable (e.g., Goldstone \& Sakamoto, 2003).

At the same time, concrete materials (or concrete representations of a to-be-learned structure) often communicate much information without communicating relevant aspects of the to-be-learned information. For example, when different numerosities are represented by a different number of frogs (as opposed to being represented by a different number of dots), the representation communicates additional, irrelevant information (e.g., shape, color, and animacy). Our goal is to examine effects of this "irrelevant concreteness" on learning and transfer. There are reasons to believe that, unlike more abstract and generic representations, such concrete and perceptually rich representations may not facilitate transfer (cf. Bassok \& Holyoak, 1989).

First, although concrete materials may be more interesting and engaging than more abstract, generic materials, the former may have limited referential flexibility. This could be because perceptually rich, concrete entities are more likely to be considered objects than symbols denoting other entities. For example, whereas it is easy to use a dot as symbol of a car, a bird, or a train, it is more difficult to use a car as a symbol of a bird. Therefore, knowledge gleaned from perceptually rich objects could be less portable than knowledge gleaned from more abstract, generic entities. As a result, the latter could be easier to transfer than the former.

These intuitions are supported by a series of studies (DeLoache, 2000; see also Uttal, Liu, \& DeLoache, 1999, for related arguments), demonstrating that while young children have no difficulty understanding that a photograph of a room is a symbol of the room, they had a much harder time understanding that the physical model of the 
room was a symbol of the room rather than an entirely different object. The results of other studies (e.g., Schwartz, 1995) indicate that pictures with high fidelity to their referents are more tightly linked to these referents than are pictures with low fidelity. Evidence also shows that children (Gentner \& Medina, 1998) and adults (Markman \& Gentner, 1993) are more likely to notice a common nonperceptual relation among objects in a scene when these objects are perceptually sparse than when they are perceptually rich.

Furthermore, in the case of irrelevant concreteness, properties of a representation that are not a part of the tobe-learned knowledge (i.e., surface features) may be erroneously interpreted as a part of the to-be-learned knowledge, thus hindering transfer (Bassok \& Olseth, 1995; Bassok, Wu, \& Olseth, 1995; Ross, 1984, 1987, 1989). Finally, there is recent evidence of possible competition between abstract and concrete representations of the same situation and that salient concrete representations may distract learners from more abstract regularities (Goldstone \& Sakamoto, 2003).

Therefore, it seems that under most conditions (i.e., outside of situations with relevant concreteness), perceptually rich, concrete representations may hinder transfer (and possibly learning) by distracting the learner from the relevant structure and/or by limiting referential flexibility of the representation. More generic, abstract materials may also in fact facilitate transfer. Our goal in this research was to investigate these possibilities. To examine transfer, we created two artificial (thus novel) and isomorphic domains. We created artificial domains (rather than using existing ones) to eliminate potential confounds stemming from differential prior knowledge, expectations, or experience across the domains.

Both domains were artificially constructed algebraic commutative groups of order three, each being isomorphic to the integers under addition modulo three. The concreteness of materials was manipulated by increasing the perceptual richness of symbols denoting entities in each domain. The first, more abstract, domain (hereafter "math") was presented to the participants as a symbolic language in which three types of symbols combine to yield a resulting symbol. The second, more concrete, domain (hereafter "science") involved interactions between three-dimensional (3-D) objects from three classes. The objects dynamically moved toward each other and merged to form a resulting object. In Experiments 1 and 2 , we examined the effects of perceptual richness on transfer or an improvement in learning and performance in one domain after learning the other domain. In Experiment 3 , we examined the effects of perceptual richness on learning.

\section{EXPERIMENT 1}

The goal of Experiment 1 was to investigate transfer of learning across the two isomorphic artificial domains. The participants learned both math and science. Half of the participants learned math first, and half learned science first. Transfer was measured by comparing average test scores on a given domain as a function of prior learning of the other domain. Test questions probed students' ability to apply learned knowledge to complex, novel problems.

\section{Method}

Participants. Thirty undergraduate students from Ohio State University participated in the experiment and received partial credit in an introductory psychology course.

Design and Materials. The experiment comprised four phases presented over $1 \mathrm{~h}$ : (1) training in domain $X,(2)$ test in domain $X$, (3) training in domain Y, and (4) test in domain Y, with the participants randomly assigned to a particular order of learning (i.e., math-then-science or science-then-math). The experiment had a 2 (domain: math vs. science) $\times 2$ (order: math-then-science vs. science-then-math) mixed design, with order as a between-subjects factor and domain as a repeated measure. Training and testing in the first domain was followed immediately by training and testing in the second domain. After testing, the participants were asked to rate the similarity of the two domains on a scale from 1 to 5 , with a rating of 1 indicating that the domains were completely different, and a rating of 5 indicating that the domains were identical.

Test scores for math and science were compared across the two conditions, math-then-science and science-then-math. Transfer due to learning of math was taken to be the difference in the average science score for math-then-science and the average science score for science-then-math. Similarly, transfer due to learning of science was taken to be the difference in the average math score for sciencethen-math and the average math score for math-then-science.

In each domain, the entities interacted according to the rules of an algebraic commutative group (these rules are presented in Table 1). The goal of training was to learn the four specific rules presented in Table 1, and properties of these rules (i.e., associativity, commutativity, and the existence of the identity element and of inverse elements), with training in both domains being isomorphic. All rules were presented one at a time and stated explicitly. For example, when a specific rule was presented in the math domain, the students were told that a combination of symbol " $\star$ " and symbol " always results in symbol " $\bullet$," with the operation being written as follows: $\star, \rightarrow \diamond$. A rule such as commutativity was not stated explicitly as "commutativity" but was rather shown as explicit examples: " $\star$, gives the same result as $\star$." In the science domain, rules were presented similarly, except that objects rather than symbols were shown (see Table 1 for examples of objects), with operations being presented as dynamic interactions of these objects. Presentation of each rule was followed by a memory check with feedback.

Testing consisted of 20 multiple-choice questions designed to measure the participants' ability to apply the learned rules to novel problems; none of the test questions used examples that were presented during training. Each test question had only one correct answer, and most of the questions required application of multiple rules. Some of the questions were significantly more complicated than those presented in the training. The following are examples of multiple-choice test questions.

(1) What can go in the blanks to make a correct statement?

$$
\ldots, \star, \ldots, \rightarrow
$$

Choose from the following: (a) and $\bullet$, (b) $\star$ and $\star$, (c) and and (d) $\bullet$ and $\star$.

(2) Find the resulting symbol:

$$
\star,
$$

(3) Do the following statements have the same results? $\rightarrow, \bullet, \bullet$ ? and $\bullet, \star \rightarrow$ ?

For both domains, the test questions were completely isomorphic and were presented in the same order. 
Table 1

Example of Stimuli and Transformation Rules Across the Two Domains

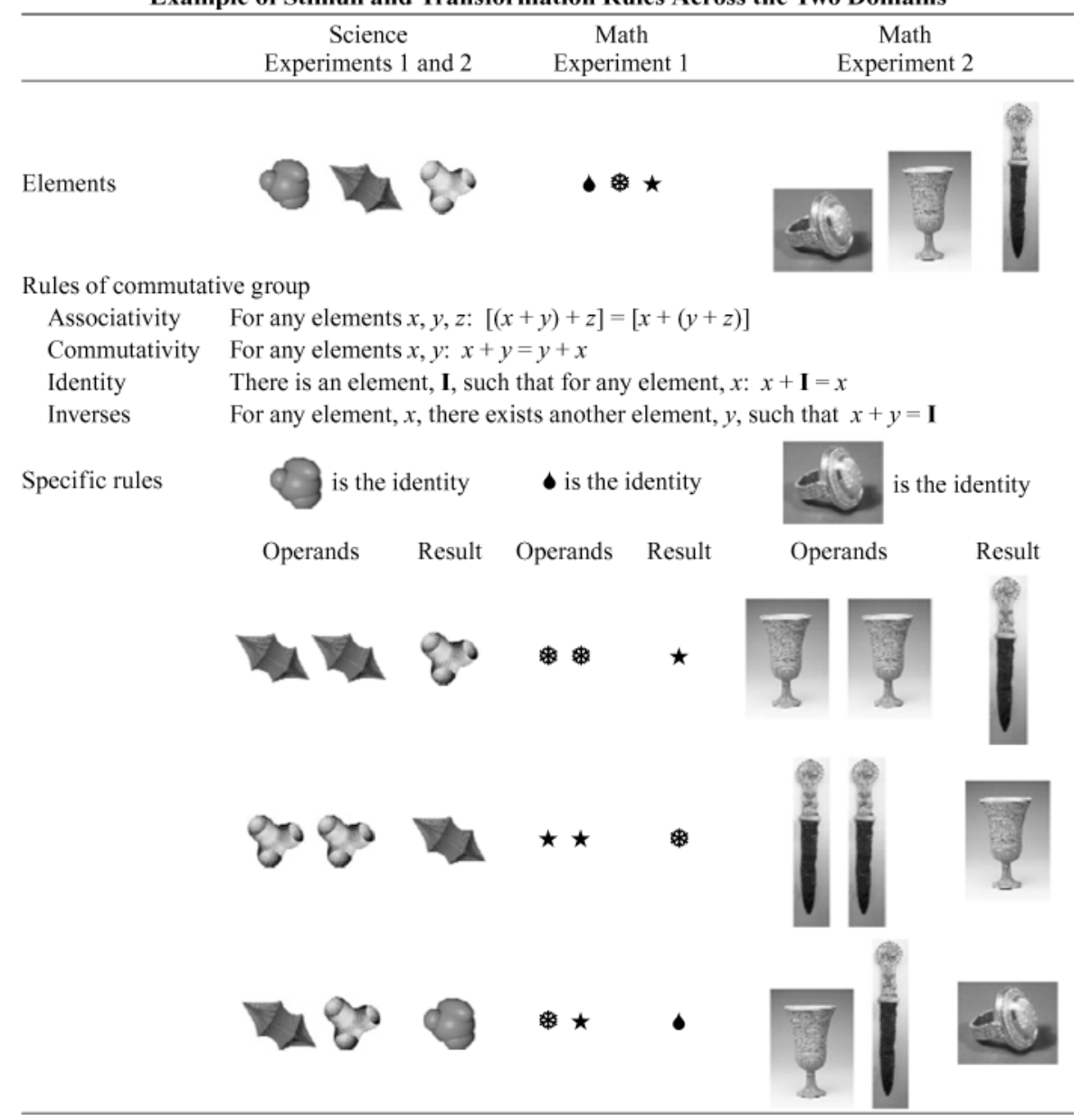

Procedure. The participants were tested individually, with all training and testing being presented on a computer screen in a selfpaced manner, with the researcher recording the participants' responses. The presentation of the two domains differed by storyline. The artificial math was presented as a symbolic language discovered on an archaeological search. Symbols of different categories combined to yield a resulting symbol. The artificial science was explained as a phenomenon observed on a planet outside of our solar system. Objects from different classes of shapes interacted to form a resulting shape. The presentation of the artificial science included movie clips demonstrating the interactions, with two or more objects coming in contact, and the interaction resulting in the emergence of a new object.

\section{Results and Discussion}

Participants' similarity ratings of the domains confirmed that the two domains were isomorphic. On a similarity scale from 1 (completely dissimilar) to 5 (structurally identical), the mean rating given by math-then-science participants was $4.6(S D=.84)$, and the mean rating given by science-then-math participants was also 4.6 $(S D=.50)$.

The data on learning and transfer across the domains are presented in the left panel of Figure 1. First, participants successfully learned in both domains $\left[M_{\text {science }}=\right.$
16.8, $\left.M_{\text {math }}=16.6\right]$, both above chance [one-sample $t \mathrm{~s}(27)>21.0, p \mathrm{~s}<.001]$. Second, there was a significant difference in performance as a function of learning order. Test scores were submitted to a two-way analysis of variance (ANOVA), with order as a factor and domain as a repeated measure. The analysis indicated a significant order $\times$ domain interaction $[F(1,26)=4.076, p=.054]$. The participants in the math-then-science condition performed significantly better on the science test than did the participants in the science-then-math condition [independent-samples $t(26)=2.6, p<.05$ ]. At the same time, there was no difference in math scores across conditions [independent-samples $t(26)<1$ ]

It could be argued, however, that the reported interaction was due to high accuracy in the math. Because participants exhibited near-ceiling accuracy in both orders of math, there was little room for improvement as a result of learning science. To examine this possibility, we performed a separate analysis on students who exhibited lower learning scores (i.e., fewer than 16 items correct) in the domain that was learned first. The results indicate that, contrary to the ceiling-effect explanation, the interaction was even more pronounced for these lower per- 


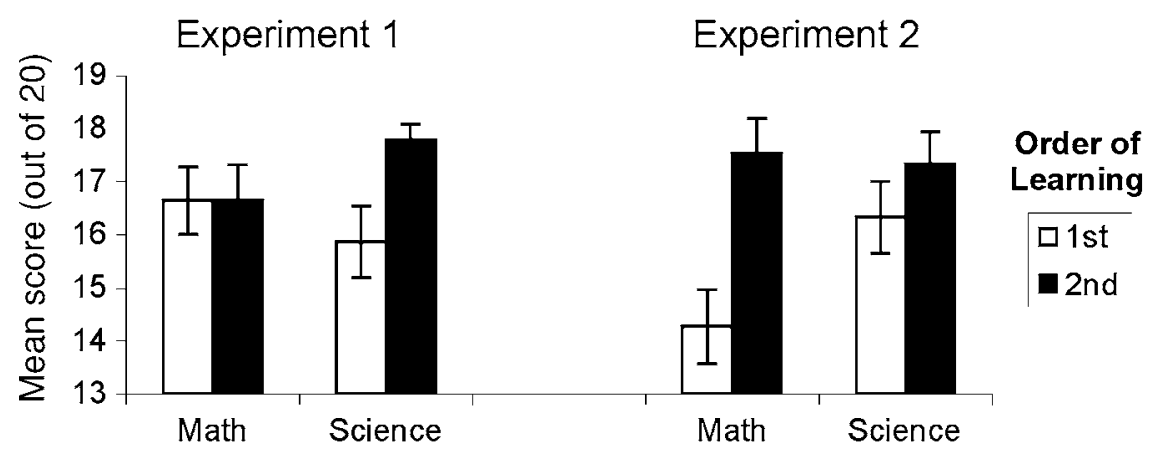

Figure 1. Mean test scores for math and science by the order of learning. Error bars represent standard errors of the mean.

forming students $[F(1,12)=17.053, p<.002]$, with no significant differences between the two orders of math $\left[M_{\text {math-then-science }}=14.6, S D=0.79\right.$ vs. $M_{\text {science-then-math }}=$ $16.3, S D=2.50$; independent-samples $t(12)=1.7, p>$ .12] and significant differences between the two orders of science $\left[M_{\text {science-then-math }}=13.9, S D=1.46\right.$ vs. $M_{\text {math-then-science }}=17.3, S D=0.76$; independent-samples $t(12)=5.51, p<.0001]$.

The higher average science score for participants in the math-then-science condition than for the sciencethen-math conditions suggests that learning of math facilitated their learning of science, whereas learning of science did not facilitate learning of math. Because both domains were novel, these data suggest that knowledge presented in a more abstract, generic format facilitates acquisition of knowledge presented in a more perceptually rich, concrete format.

It could be counterargued, however, that the observed differential transfer may stem from other differences between the domains. In particular, there was a difference in cover story; and more important, science was presented in a dynamic format, whereas math was presented in a static format.

This issue was addressed in Experiment 2. In particular, if the observed differences in learning stem from the concreteness of materials, rather than from extraneous factors, introducing math in a more concrete format than science should lead to an attenuation or a reversal of the differential transfer effects found in Experiment 1.

\section{EXPERIMENT 2}

\section{Method}

Participants. Thirty undergraduate students from Ohio State University participated in the experiment and received partial credit in an introductory psychology course.

Materials, Design, and Procedure. The materials used were identical to those of Experiment 1, with one critical difference; math symbols were replaced by images of 3-D objects, as is shown in Table 1. Each image was a specific identifiable object, and therefore math materials in Experiment 2 were more concrete than science materials, which were unfamiliar objects. The design and procedure of the experiment were identical to those of Experiment 1.

\section{Results and Discussion}

As in Experiment 1, students in both learning order conditions noticed the similarity between the two domains, with the average similarity rating in the math-then-science condition being $4.3(S D=.62)$, and the average similarity rating for the science-then-math condition being 4.7 $(S D=.46)$, with no significant differences between the conditions.

Test scores by conditions are presented in the right panel of Figure 1. As in Experiment 1, students successfully learned in both domains $\left(M_{\text {science }}=16.8, M_{\text {math }}=\right.$ $15.9)$, both above chance $(p \mathrm{~s}<.001)$. However, this time, the learning of math first was less successful than the learning of science first. Furthermore, a reversal of transfer from Experiment 1 was found (see the right panel in Figure 1): Unlike in Experiment 1, where learning of math facilitated learning of science, in this experiment, learning of science facilitated learning of math, with math scores in the science-then-math condition being higher than those for students in the math-thenscience condition.

These results were supported by an order $\times$ domain mixed ANOVA, which revealed a significant domain $X$ order interaction $[F(1,28)=22.055, p<.001]$, with no significant differences between the two science orders [independent-samples $t(28)=1.1, p>.27$ ] and significant differences between the two math orders [independentsamples $t(28)=3.428, p<.01]$. Furthermore, there was a significant effect of domain $[F(1,28)=4.22, p<.05]$, with participants in the math condition (which used perceptually rich symbols) exhibiting lower test scores than participants in the science condition, thus suggesting that the use of perceptually rich symbols may negatively affect learning.

The results of Experiments 1 and 2 indicate that transfer of learning is facilitated when initial learning in the base domain involves objects that are more abstract and generic than those of the target domain. Furthermore, the results suggest that the concreteness of objects hinders not only transfer, but learning itself. To examine the effects of concreteness on learning, we conducted Experiment 3. 


\section{EXPERIMENT 3}

\begin{abstract}
Method
Participants. Eighty-one undergraduate students from Ohio State University participated in the experiment for partial credit in an introductory psychology course.

Materials, Design, and Procedure. The story line and training were identical to those used in the math condition of Experiments 1 and 2. However, the symbols varied across conditions, as shown in Table 2 . The participants were randomly assigned to one of four conditions: (1) perceptually sparse black symbols, (2) perceptually rich correlated symbols, (3) perceptually rich uncorrelated symbols, and (4) perceptually rich real objects. The perceptually rich, correlated and uncorrelated symbols had four dimensions: shape (which was the determiner of their category membership), outline color, interior pattern, and interior pattern color. In the perceptually rich, correlated condition, color and pattern of shading of symbols correlated with their shape (e.g., circles were always red, whereas diamonds were green). In the uncorrelated condition, the relevant feature of a symbol was its shape, and the color and pattern of shading varied independently (see Table 2). The perceptually rich, correlated and uncorrelated conditions were included to investigate whether random variance makes learning difficult (in which case the correlated condition should elicit better learning than the uncorrelated condition) or whether perceptual richness itself makes learning difficult (in which case there should be little or no difference between the correlated and uncorrelated conditions). The real objects were similar to those used in Experiments 1 and 2 (see Table 2 for examples). The training and testing procedures were identical to those used in Experiments 1 and 2.
\end{abstract}

\section{Results and Discussion}

Learning scores are presented in Figure 2. First, students in all conditions learned the material, exhibiting above-chance performance at test (all $p$ s $<.001$ ). More important, there were significant differences among the conditions $[F(3,65)=3.85, p<.05]$, with participants in the black symbol condition performing significantly better than those in the uncorrelated condition or the real objects condition (post hoc Tukey test, all $p$ s $<.05$ ) and marginally better than those in the correlated condition $(p<.065)$. At the same time, there was no difference among the perceptually rich conditions (post hoc Tukey test, all $p \mathrm{~s}>$.9). The latter two findings are of utmost importance, indicating that perceptual richness hindered learning.

\section{GENERAL DISCUSSION}

The three reported experiments reveal several key regularities. Experiment 1 indicated that transfer of learning between two isomorphic domains (e.g., performance in the second domain after learning of the first domain) was greater when the first domain used more abstract, generic representations than when it used perceptually rich, concrete representations. Experiment 2 replicated these findings, while introducing some additional controls. Experiment 3 indicated that the use of perceptually rich, concrete materials hinders learning. Overall, results of the three reported experiments present novel findings indicating that irrelevant concreteness of study materials negatively affects both learning and transfer.

Note that recent evidence (Goldstone \& Sakamoto, 2003 ) indicates that perceptually rich, concrete representations facilitate learning of complex principles. However, Goldstone and Sakamoto used concrete representations communicating relevant aspects of the to-be-learned information (i.e., active agents were represented as ants, and stationary resources were represented as apples, as opposed to a more abstract representation, in which both agents and resources were represented as dots). Unlike Goldstone and Sakamoto's experiments, our research used concrete materials that did not communicate relevant aspects of the to-be-learned information. Taken together, results of Goldstone and Sakamoto and those of the present experiments indicate that in order to facilitate learning, concrete representations have to capture important aspects of to-be-learned knowledge; otherwise, concrete representations would hinder, rather than facilitate, learning.

Several factors can account for these effects of irrelevant concreteness on learning and transfer. First, concrete representations may automatically engage the perceptual system while preventing deeper conceptual processing. Furthermore, perceptually rich, concrete representations can increase dissimilarity between entities in both domains, thus limiting structural alignment, which could be important for the extraction of underlying relational commonalities: Some evidence shows that perceptually sparse representations are more likely to emphasize a

Table 2

Examples of Symbols Used Across Conditions in Experiment 3

\begin{tabular}{cccc}
$\begin{array}{c}\text { Perceptually Sparse } \\
\text { Symbols }\end{array}$ & $\begin{array}{c}\text { Perceptually Rich } \\
\text { Symbols } \\
\text { (Features are correlated) }\end{array}$ & $\begin{array}{c}\text { Perceptually Rich } \\
\text { Symbols } \\
\text { (Features are uncorrelated) }\end{array}$ & $\begin{array}{c}\text { Perceptually Rich } \\
\text { Symbols } \\
\text { (Real objects) }\end{array}$ \\
\hline & & &
\end{tabular}




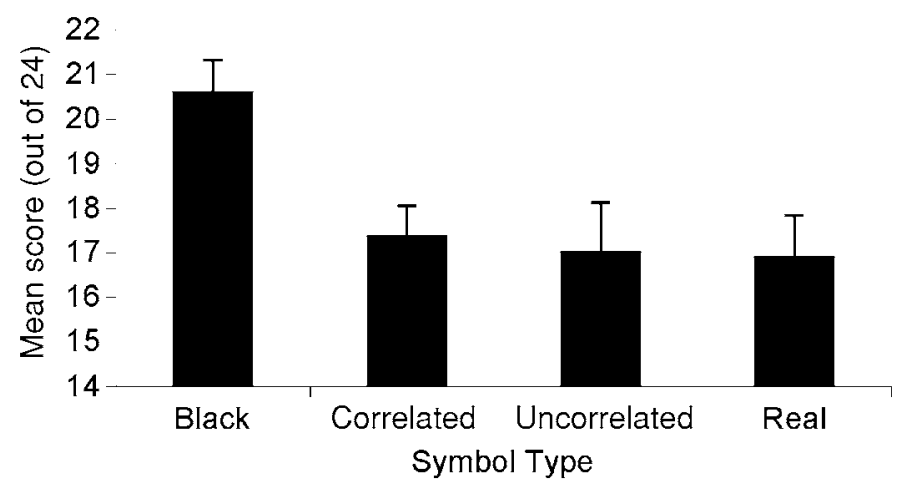

Figure 2. Mean test scores for math in Experiment 3. Error bars represent standard errors of the mean.

nonperceptual relation among entities than perceptually rich representations (Gentner \& Medina, 1998; Gentner \& Rattermann, 1991; Markman \& Gentner, 1993).

Second, irrelevant aspects of a concrete representation could be erroneously interpreted as a part of the to-belearned knowledge (Bassok \& Olseth, 1995; Bassok, Wu, \& Olseth, 1995; Ross, 1984, 1987, 1989). Finally, concrete objects may have limited referential flexibility, because they are more likely to be interpreted as entities than as symbols (DeLoache, 2000; Uttal, Liu, \& DeLoache, 1999; see also Goldstone \& Sakamoto, 2003), and as a result, participants may fail to apply knowledge learned about more concrete entities to more abstract entities.

The fact that irrelevant concreteness was found to hinder both learning and transfer may have important implications for our understanding of learning of complex domains, specifically that of mathematics and science. The dominant view in the educational community has been that perceptually rich, concrete, and entertaining materials are useful for acquisition of knowledge and transfer of this knowledge outside the learned situations (Ball, 1992; Cobb, Yackel, \& Wood, 1992). Our research suggests that although intuitively appealing, this view may be very limited. In order to facilitate learning, perceptually rich, concrete representations must communicate relevant aspects of the to-be-learned information. However, even then there might be a tradeoff between learning and transfer (cf. Goldstone \& Sakamoto, 2003). In addition, the results of the present research indirectly suggest that learning of mathematics, which is populated by abstract entities represented by generic symbols, can facilitate learning of science, which is populated by more perceptually rich, concrete entities.

\section{REFERENCES}

BALL, D. L. (1992). Magical hopes: Manipulatives and the reform of math education. American Educator, 16, 14-18.

BassoK, M., \& HolyOAK, K. J. (1989). Interdomain transfer between isomorphic topics in algebra and physics. Journal of Experimental Psychology: Learning, Memory, \& Cognition, 15, 153-166.

BAssoK, M., \& Olseth, K. L. (1995). Object-based representations: Transfer between cases of continuous and discrete models of change.
Journal of Experimental Psychology: Learning, Memory, \& Cognition, 21, 1522-1538.

BassoK, M., Wu, L.-L., \& Olseth, K. L. (1995). Judging a book by its cover: Interpretative effects of context on problem-solving transfer. Memory \& Cognition, 23, 354-367.

CobB, P., YACKel, E., \& Wood, T. (1992). A constructivist alternative to the representational view of mind in mathematics education. Journal for Research in Mathematics Education, 23, 2-33.

DeLoache, J. S. (2000). Dual representation and young children's use of scale models. Child Development, 71, 329-338.

DetTerman, D. K. (1993). The case for the prosecution: Transfer as an epiphenomenon. In D. K. Detterman \& R. J. Sternberg (Eds.), Transfer on trial: Intelligence, cognition, and instruction (pp. 1-24). Westport, CT: Ablex.

DiEnES, Z. P. (1960). Building up mathematics. London: Hutchinson.

Gentner, D., \& Medina, J. (1998). Similarity and the development of rules. Cognition, 65, 263-297.

Gentner, D., \& Rattermann, M. J. (1991). Language and the career of similarity. In S. A. Gelman \& J. P. Byrnes (Eds.), Perspectives on thought and language: Interrelations in development (pp. 225-277). Cambridge: Cambridge University Press.

Goldstone, R. L., \& Sakamoto, Y. (2003). The transfer of abstract principles governing complex adaptive systems. Cognitive Psychology, 46, 414-466.

Howard, P., Perry, B., \& Tracey, D. (1997). Mathematics and manipulatives: Comparing primary and secondary teachers' views. Paper presented at the annual conference of the Australian Association for Research in Education, Brisbane.

Markman, A. B., \& GentNer, D. (1993). Structural alignment during similarity comparisons. Cognitive Psychology, 25, 431-467.

Ross, B. H. (1984). Remindings and their effects in learning a cognitive skill. Cognitive Psychology, 16, 371-416.

Ross, B. H. (1987). This is like that: The use of earlier problems and the separation of similarity effects. Journal of Experimental Psychology: Learning, Memory, \& Cognition, 13, 629-639.

Ross, B. H. (1989). Distinguishing types of superficial similarities: Different effects on the access and use of earlier problems. Journal of Experimental Psychology: Learning, Memory, \& Cognition, 15, 456-468.

SchWARTZ, D. L. (1995). Reasoning about the referent of a picture versus reasoning about the picture as the referent: An effect of visual realism. Memory \& Cognition, 23, 709-722.

Uttal, D. H., LiU, L. L., \& DeLoache, J. S. (1999). Taking a hard look at concreteness: Do concrete objects help young children learn symbolic relations? In L. Balter \& C. Tamis-LeMonda (Eds.), Child psychology: A handbook of contemporary issues (pp. 177-192). Philadelphia: Psychology Press.

(Manuscript received April 19, 2004; revision accepted for publication July 26, 2004.) 